\title{
Odontoma composto associado canino incluso no palato: relato de caso
}

\author{
Compound odontoma associate with canine included in palate: case report \\ Odontoma compuesto asociado al canino en el paladar: reporte de un caso \\ Breno dos Reis FERNANDES ${ }^{1}$ \\ Darah Ligia MARCHIORI ${ }^{2}$ \\ Daniel de Lima e Sá MEDRONHO ${ }^{3}$ \\ Gabriel MULINARI-SANTOS ${ }^{\mathbf{1}}$ \\ Patrick Peloso Pereira FIGUEIRA ${ }^{3}$ \\ Henrique HADAD ${ }^{1}$ \\ Emmanuel Pereira ESCUDEIRO \\ Jonathan Ribeiro da SILVA ${ }^{3}$ \\ ${ }_{1}^{1}$ Faculdade de Odontologia de Araçatuba, UNESP-Univ. Estadual Paulista, Araçatuba-SP, 16015-050 Araçatuba-SP, Brasil \\ Universidade Alto Vale do Rio do Peixe, UNIARP - Campus Caçador, 89500-000 Caçador-SC, Brasil \\ ${ }^{3}$ Hospital das Clínicas de Teresópolis Constantino Ottaviano HCTCO, 25976-016 Teresópolis-RJ, Brasil
}

\section{Resumo}

Odontoma refere-se a tumores de origem odontogênica. Sua etiologia envolve distúrbios de desenvolvimento, traumatismos e infecções. Embora o crescimento seja lento e geralmente assintomático, complicações de ordem estética e funcional podem advir da permanência desta lesão. Os odontomas podem ser classificados como complexos e compostos, e o seu tratamento envolve a remoção cirúrgica conservadora com posterior exame histopatológico. Neste artigo é relatado um caso clínico de uma remoção de um canino incluso no palato associado a um odontoma com posterior enxertia utilizando enxerto do ramo mandibular particulado.

Descritores: Odontoma; Cirurgia Bucal; Dente Canino.

\section{Abstract}

Odontoma refers to tumors of odontogenic origin. Its etiology involves developmental disorders, injuries and infections. Although growth is slow and usually asymptomatic, aesthetic and functional complications may result from the permanence of this lesion. Odontomas can be classified as complex and compound, and their treatment involves conservative surgical removal with subsequent histopathological examination. This paper reports a case report of a canine removal included in the palate associated with a posterior graft odontoma using graft of the particulate mandibular ramus.

Descriptors: Odontoma; Surgery, Oral; Cuspid.

\section{Resumen}

Odontoma se refiere a tumores de origen odontogénico. Su etiología implica trastornos del desarrollo, lesiones e infecciones. Aunque el crecimiento es lento y generalmente asintomático, la permanencia de esta lesión puede producir complicaciones estéticas y funcionales. Los odontomas se pueden clasificar como complejos y compuestos, y su tratamiento consiste en la extirpación quirúrgica conservadora con un examen histopatológico posterior. Este documento presenta un informe de un caso de extracción de caninos incluido en el paladar asociado con un odontoma de injerto posterior que utiliza un injerto de la rama mandibular mandibular particulada.

Descriptores: Odontoma; Cirugía Bucal; Diente Canino.

\section{INTRODUÇÃO}

Os odontomas são os tipos mais comuns dos tumores odontogénicos, pois são considerados mais como uma anomalia de desenvolvimento do que uma neoplasia verdadeira ${ }^{1-3}$.

De acordo com a Organização Mundial de Saúde (OMS), os odontomas podem ser classificados em dois tipos principais: complexo e composto. Os odontomas compostos são aqueles que se originam de uma proliferação exagerada da lâmina dentária, em que todos os tecidos dentais estão representados de uma maneira organizada, formando estruturas semelhantes a dentículos. Nos odontomas complexos, por sua vez, os tecidos dentais representados estão desordenados, ou seja, morfologicamente não remetem à forma de dentes ${ }^{4}$.

Estas lesões são geralmente assintomáticas, sendo identificadas em exames radiográficos de rotina. Achados clínicos, como impactação, mal posicionamento, anodontia, malformação e desvitalização de dentes, adjacentes podem estar associados aos odontomas ${ }^{5,6}$.

Radiograficamente, os odontomas compostos apresentam-se como um conjunto de estruturas semelhantes aos dentes, de forma e tamanho variáveis. Já os odontomas complexos aparecem como massas radiopacas amorfas envolvidas por estreita zona radiolúcida ${ }^{7,8}$.
O tratamento para os odontomas é sua total excisão cirúrgica, com prognóstico bastante favorável, sendo raros os casos de recidiva, e a reparação óssea, realizada com certa facilidade?

\section{CASO CLÍNICO}

Paciente do sexo masculino, 52 anos, leucodema, compareceu no ambulatório de Cirurgia e Traumatologia Bucomaxilofacial do Hospital das Clínicas de Teresópolis/RJ queixando-se de ausência do elemento dentário 23. Ao exame físico o mesmo apresentava ausência do elemento 23. Foi solicitada tomografia computadorizada para analisar o caso, no qual era possível visualizar o dente 23 incluso no palato e um odontoma composto (Figuras 1 e 2).

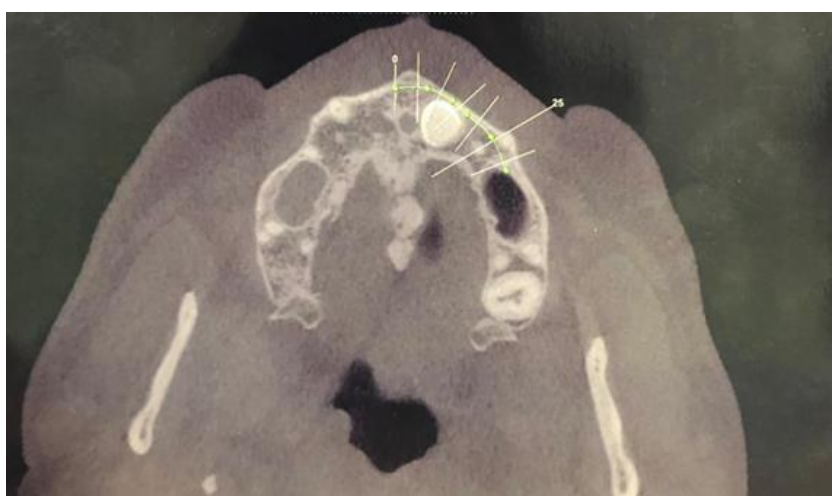

Figura 1: Tomografia computadorizada com dente 23 incluso no palato. 


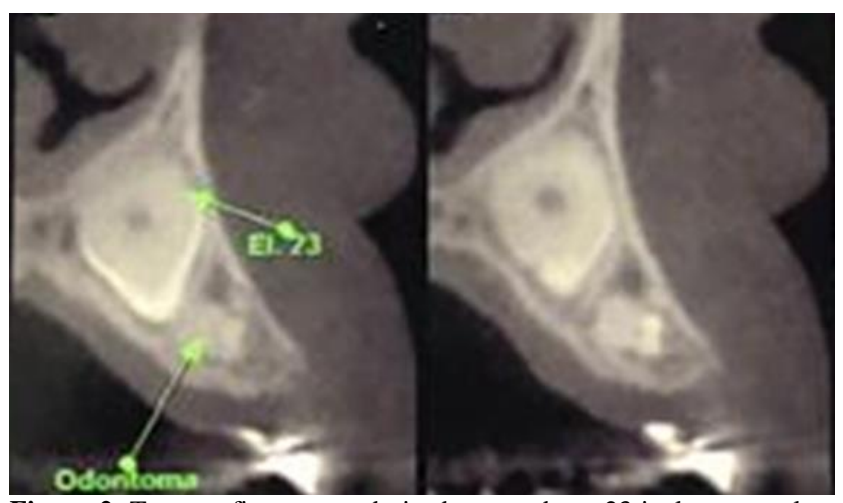

Figura 2: Tomografia computadorizada com dente 23 incluso no palato e odontoma composto.

Diante os achados clínicos a hipótese de diagnóstico foi odontoma associado ao canino incluso. $\mathrm{O}$ paciente foi submetido à cirurgia sob anestesia local para remoção do elemento e do tumor. Primeiramente foi realizada incisão intrasulcular na região do palato seguido do deslocamento do tecido para visualização direta dos elementos (Figura 3). No primeiro passo da cirurgia o odontoma foi removido com auxilio de extrator bucal (Figura 4).

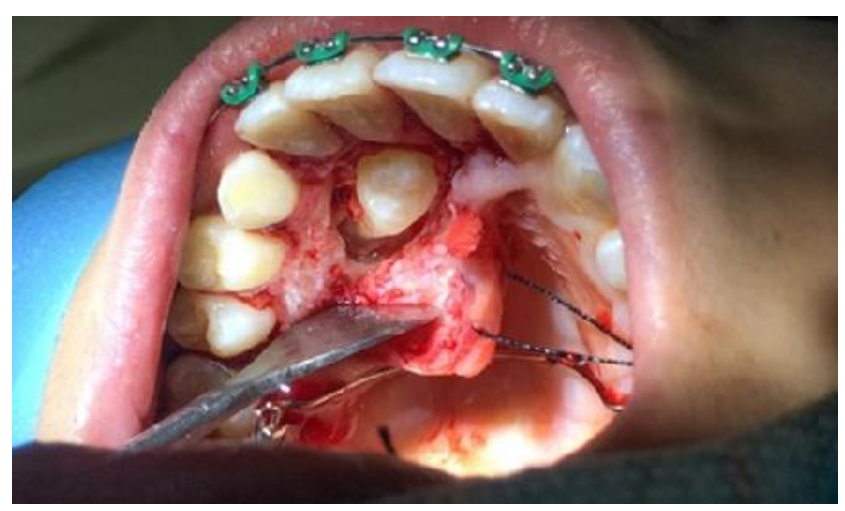

Figura 3: Incisão intrasulcular na região do palato seguido do deslocamento do tecido para visualização direta do dente incluso.

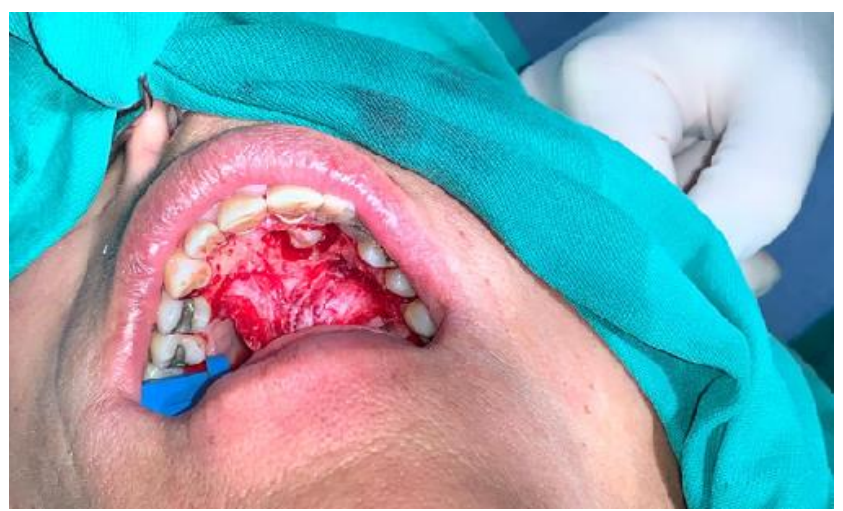

Figura 4: No primeiro passo da cirurgia o odontoma foi removido com auxilio de um extrator bucal.

Na sequência, com auxílio de caneta de alta rotação e broca 702, foi realizada ostectomia e odontosecção do elemento dentário (Figura 5).

Após a remoção do canino e do odontoma, o paciente acabou perdendo um pouco de osso na região do palato, causando um pequeno afundamento. Diante disso como já havia sido elaborado no planejando pré-operatório, foi realizado um acesso intraloral em linha obliquoa da mandíbula para acessar o ramo mandibular (Figura 6) e um enxerto de $2 \mathrm{~cm}$ foi removido do local (Figura 7), posteriormente o mesmo foi particulado através de um triturador para osso (Figura 8) e logo em seguida foi inserido na aloja cirúrgica. (Figura 9), e realizado a sutura no local utilizando fio nylon 5-0. O paciente foi de alta recebendo alta pela especialidade para acompanhamento ambulatorial, com prescrição de: ibuprofeno $600 \mathrm{mg}$ a cada 8 horas por 3 dias, dipirona 500mg a cada 6 horas por 05 dias, além de amoxicilina 500mg a cada 8 horas por 07 dias, mantido acompanhamento ambulatorial semanal por 8 semanas, mensal por 3 meses, e após apenas semestral. Hoje o paciente apresenta 06 messes de pós-operatório sem nenhuma queixa álgicas, estética e funcional.

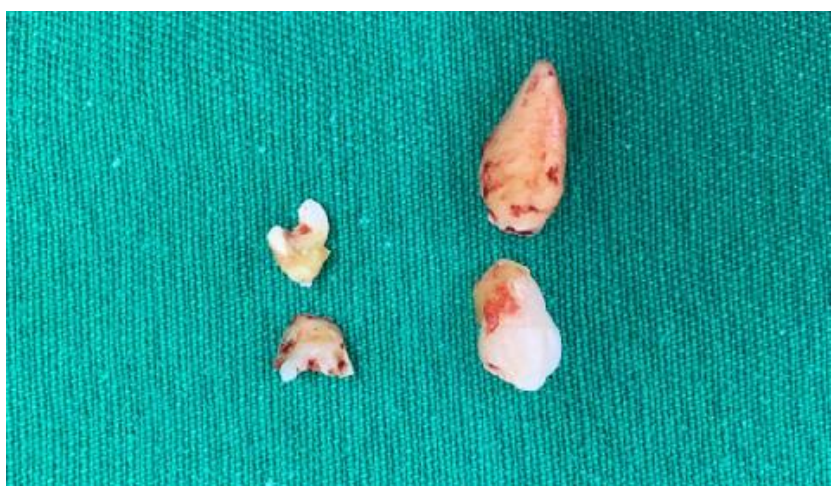

Figura 5: Fragmentos do canino removido.

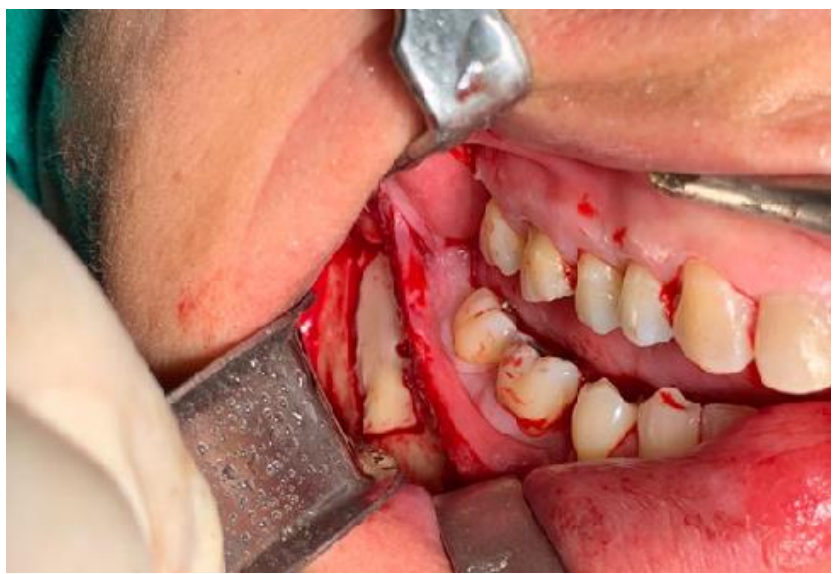

Figura 6: Fragmentos do canino removido.

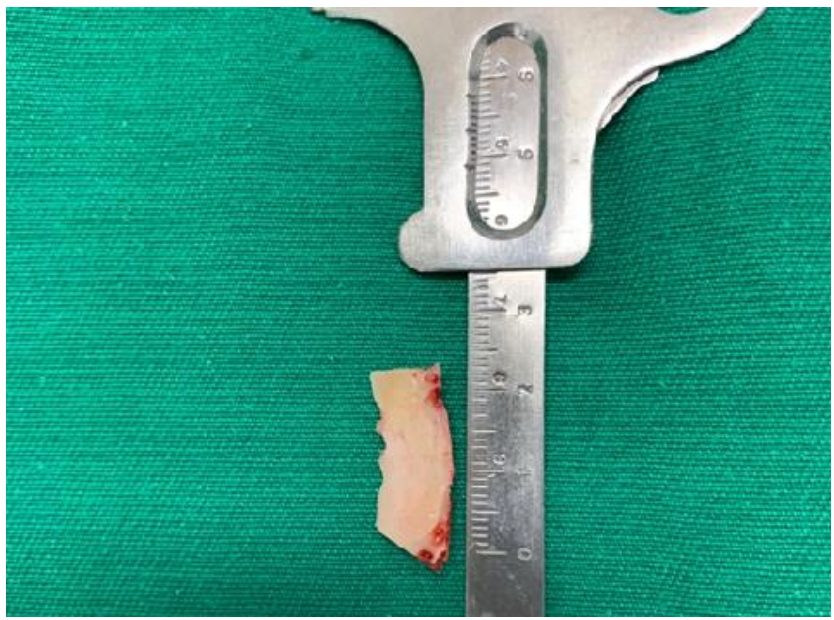

Figura 7: Enxerto de ramo mandibular de $2 \mathrm{~cm}$ foi removido. 


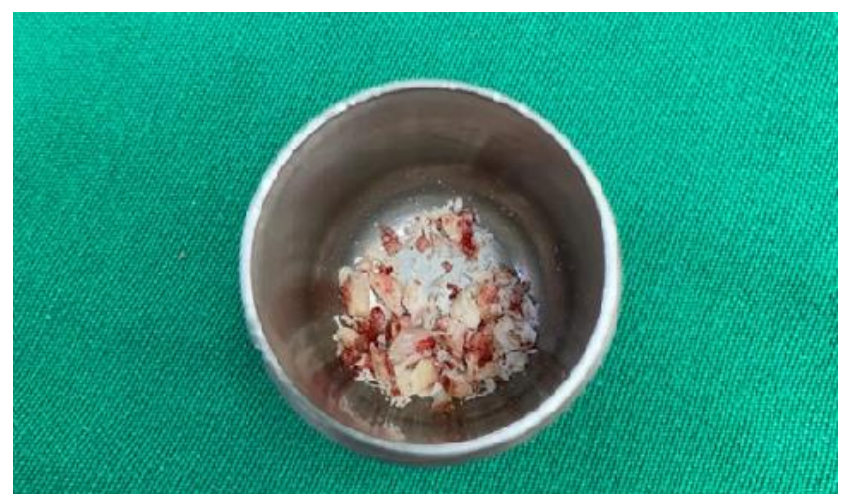

Figura 8: O osso foi particulado através de um triturador para osso.

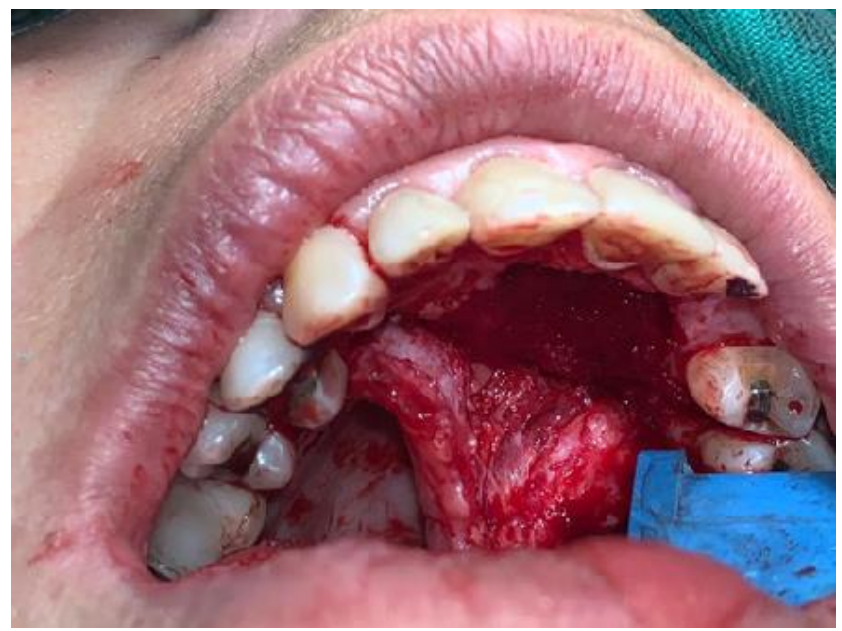

Figura 9: O osso foi inserido na loja cirúrgica.

Como o paciente havia perdido parte da gordura orbital, aproveitando o acesso vestibular maxilar realizado intraoral, foi removido parte do corpo adiposo da bochecha, para auxiliar no ganho de conteúdo orbitário (Figura 9). Após a reconstrução os tecidos profundos foram suturados com Vycril $3.0 \mathrm{e} \mathrm{a}$ pele com Nylon 5.0. Após 02 dia de pós-operatório o paciente havia apresentado melhoras significativa na enoftalmia além da ausência de diplopia recebendo alta pela especialidade para acompanhamento ambulatorial, com prescrição de: ibuprofeno $400 \mathrm{mg}$ e dipirona 500mg a cada 6 horas por 03 dias, além de amoxicilina 500mg a cada 8 horas por 07 dias. No sétimo dia de pós-operatório foi realizado a remoção de sutura, e mantido acompanhamento ambulatorial semanal por 8 semanas, mensal por 3 meses, e após apenas semestral. Hoje o paciente apresenta 06 messes de pós-operatório sem intercorrências.

\section{DISCUSSÃO}

Os odontomas são patologias assintomáticas, de evolução lenta, atingindo, na maioria dos casos, pequenas proporções ${ }^{10}$. Na maioria dos casos, os odontomas são encontrados em pacientes jovens. Muitos autores relataram que a idade média no momento do diagnóstico é em torno dos 14 anos, embora possam ser observados ocasionalmente em qualquer idade e sem predileção por sexo ${ }^{1}$. A presença dos odontomas é uma das causas importantes de retenção dental, sendo geralmente diagnosticados em exames radiográficos de rotina ou quando se procura investigar a causa da não erupção de um dente permanente ${ }^{11}$. Radiograficamente, os odontomas compostos apresentam-se como um conjunto de estruturas semelhantes aos dentes, de forma e tamanho variáveis, já os odontomas complexos aparecem como massas radiopacas amorfas, envolvidas também por uma estreita zona radiolúcida $^{7,8}$. O tratamento de escolha foi ao encontro do relatado na literatura, optando-se pela excisão cirúrgica, que é o tratamento mais preconizado para os casos de odontoma ${ }^{12}$.

\section{CONSIDERAÇÕES FINAIS}

O odontoma composto é uma malformação benigna relativamente comum e de fácil diagnóstico clínico e por imagens. Seu tratamento de escolha consiste na excisão cirúrgica, na qual deve ser realizada a completa remoção e curetagem tão logo sejam diagnosticados, a fim de promover a reabilitação e evitar possíveis fraturas indesejáveis.

\section{REFERÊNCIAS}

1. Teruhisa U, Murakami J, Hitasomi M, Yanagi Y, Asaumi J. A case of unerupted lower primary second molar associated with compound odontoma. Open Dent J. 2009;3:173-76.

2. Ladeinde AL, Ajayi OF, Ogunlewe MO, Adeyemo WL, Arotiba GT, Bamgbose BO et al. Odontogenic tumors: a review of 319 cases in a Nigerian teaching hospital. Oral Surg Oral Med Oral Pathol Oral Radiol Endod. 2005;99(2):191-95.

3. Hidalgo-Sánchez O, Leco-Berrocal MI, MartínezGonzáles JM. Metaanalysis of the epidemiology and clinical manifestations of odontomas. Med Oral Patol Oral Cir Bucal. 2008;13(11):730-34.

4. Pires LD, Krüger MLB, Viana ES, Kramer PF, Ferreira SL. Odontoma: estado da arte e relato de caso clínico. Stomatos. 2007;13(24):21-9.

5. Chang JY, Wang JT, Wang YP, Liu BY, Sun A, Chiang CP. Odontoma: a clinicopathologic study of 81 cases. J Formos Med Assoc. 2003; 102(12):876-82.

6. Hisatomi M, Asaumi JI, Konouchi H, Honda Y, Wakasa T, Kishi K. A case of complex odontoma associated with an impacted lower deciduous second molar and analysis of the 107 odontomas. Oral Dis. 2002;8(2):100-5.

7. Sheehy EC, Odell EW, Al-Jaddir G. Odontomas in the primary dentition: literature review and case report. J Dent Child (Chic). 2004;71(1):73-6.

8. Sasaki PS, Biancalana H, Duarte DA. Odontoma em pacientes odontopediátricos: repercussöes clínicas e proposiçäo de tratamento Rev Assoc Paul Cir Dent. 2002;56(5):382-86.

9. Serra-Serra G, Berini-Aytés L, Gay-Escoda C. Erupted odontomas: a report of three cases and review of the literature. Med Oral Patol Oral Cir Bucal. 2009;14(6):299-303.

10. Cardoso LC, Miyahara GI, Magro Filho O, Garcia 
Junior IR, Soubhia AMP. Odontoma combinado associado a dentes não-irrompidos: relato de casos clínicos. Rev Odontol Araçatuba. 2003;24:47-51.

11. Lukes SM, Wachter KM. Compound odontoma: a case study. J Dent Hyg. 2003;77(1):47-9.

12. Chrcanovic RB, Jaeger F, Freire-Maya B. Twostage surgical removal of large complex odontoma. Oral Maxillofac Surg. 2010; 14(4):247-52.

\section{CONFLITO DE INTERESSES}

Os autores declaram não haver conflitos de interesse.

\section{AUTOR PARA CORRESPONDENCIA}

Gabriel Mulinari dos Santos

gabriel_mulinari@hotmail.com

Submetido em 30/07/2019

Aceito em 21/11/2019 